Pure and Applied Mathematics Quarterly

Volume 9, Number 3

$417-435,2013$

\title{
Visualization of 2-Dimensional Ricci Flow
}

\author{
Junfei Dai, Wei Luo*, Min Zhang, Xianfeng Gu and Shing-Tung Yau
}

\begin{abstract}
The Ricci flow is a powerful curvature flow method, which has been successfully applied in proving the Poincaré conjecture. This work introduces a series of algorithms for visualizing Ricci flows on general surfaces.

The Ricci flow refers to deforming a Riemannian metric on a surface proportional to its Gaussian curvature, such that the curvature evolves like a heat diffusion process. Eventually, the curvature on the surface converges to a constant function. Furthermore, during the deformation, the conformal structure of the surfaces is preserved. By using Ricci flow, any closed compact surface can be deformed into either a round sphere, a flat torus or a hyperbolic surface.

This work aims at visualizing the abstract Ricci curvature flow partially using the recent work of Izmestiev [8]. The major challenges are to represent the intrinsic Riemannian metric of a surface by extrinsic embedding in the three dimensional Euclidean space and demonstrate the deformation process which preserves the conformal structure. A series of rigorous and practical algorithms are introduced to tackle the problem. First, the Ricci flow is discretized to be carried out on meshes. The preservation of the conformal structure is demonstrated using texture mapping. The curvature redistribution and flow pattern is depicted as vector fields and flow fields on the surface. The embedding of convex surfaces with prescribed Riemannian metrics is illustrated by embedding meshes with given edge lengths. Using the variational principle developed by Izemestiev, the embedding is realized by optimizing a special energy function, the unique optimum is the solution.
\end{abstract}

Received February 15, 2013.

${ }^{*}$ Corresponding author. 
418 Junfei Dai, Wei Luo, Min Zhang, Xianfeng Gu and Shing-Tung Yau

Ricci flow can serve as a novel tool for visualization. It has broad applications, such as surface parameterization, shape design by curvature, shape deformation and shape analysis.

Keywords: Ricci Flow, Poincaré conjecture, uniformization metric, curvature, metric, surface parameterization.

\section{INTRODUCTION}

The Poincaré conjecture claims any closed, simply connected 3-manifold is homeomorphic to the 3 -sphere. This is the corner stone for modern topology. It has been recently solved by the Ricci flow method.

The Ricci flow refers to deforming the Riemannian metric on the manifold according to the curvature, such that the curvature evolves according to a heat diffusion process. Eventually, the curvature on the manifold converges to a constant function.

The Ricci flow has become a powerful tool in geometry and topology, it also has broad applications in engineering fields, such as surface parameterization in graphics, manifold spline in geometric modeling. Although the abstract concept of the Ricci flow is difficult to convey, visualization techniques will help us to see how the Ricci flow acts. The major challenges for visualizing the profound concepts related to Ricci flow are as follows:

(1) General 3-manifolds can not be embedded in $\mathbb{R}^{3}$, even for the simplest one, the 3 -sphere, just like a closed sphere can not be embedded in the plane.

(2) The highly non-linear Ricci flow was invented for smooth manifolds, most computational techniques in visualization are based on discrete representations.

(3) During the surface Ricci flow, the metric is deformed but the conformal structure is preserved. The concept of conformal structure is abstract and invisible.

(4) The curvature and the Riemannian metrics are intrinsic in nature, perception mainly depends on extrinsic embedding. Even for surfaces, there exists no general method to isometrically embedding a surface with a prescribed metric. 
We tackle these challenges using the following algorithms. First, we focus on Ricci flows on surfaces instead of general three dimensional manifolds. The Ricci flow is discretized to be carried out on meshes, based on the discrete Ricci flow theory and the computational methods. The preservation of the angle structure (conformal structure) is demonstrated using texture mappings, which have prominent angular features. The curvature redistribution and flow pattern is depicted as vector fields and flow fields on a surface. The embedding of convex surfaces with prescribed Riemannian metrics is illustrated by embedding meshes with given edge lengths using Izmestiev's work.

This work has the following major contributions

(1) A set of algorithms to visualize intrinsic Ricci flow on surfaces, and the conformal deformation of the metrics.

(2) Algorithm for visualizing conformal structures, conformal invariants for general surfaces.

(3) A novel variational algorithm for embedding convex surfaces in $\mathbb{R}^{3}$ by their metric based on Izmestiev's work.

(4) A rigorous and practical algorithm for designing convex shapes by Gaussian curvature.

\section{Previous Work}

R. Hamilton introduced the concept of surface Ricci flow in [7]. In [3], theoretical results of combinatorial Ricci flow have been established. Contrast with the deficiency of conventional Ricci flow, the authors introduced efficient algorithms in [9] for computing hyperbolic structures and real projective structures on surfaces using discrete hyperbolic Ricci flow.

There are many applications in computer graphics and geometric modeling directly related with computing a desired Riemannian metric with prescribed Gaussian curvature on a given surface, a direct application is surface parameterization. A lot of researches have been done on mesh parameterization due to their applications in computer graphics. The surveys of $[4,11]$ provide excellent reviews on various kinds of mesh parameterization techniques. Ricci flow can also be applied for manifold spline $[6,5]$.

Circle packing was first introduced by Thurston in his work on geometry and topology of three-manifolds [13]. A practical software system for circle packing 
420 Junfei Dai, Wei Luo, Min Zhang, Xianfeng Gu and Shing-Tung Yau

with improved algorithm can be found in [12], which considers combinatorial structures of the triangulation only. Recently, circle packing has been generalized to circle patterns $[1,2]$ and used for surface parameterization in [10], which is in Euclidean geometry.

In order to visualize Ricci curvature lows on surfaces, we use image based flow visualization method [14].

\section{BACKGROUND}

\subsection{Ricci Flow for Smooth Manifolds.}

Ricci Flow on Surfaces. Suppose $S$ is a smooth surface embedded in $\mathbb{R}^{3}$, therefore $S$ has an induced Riemannian metric $\mathbf{g}$. Suppose $u: S \rightarrow \mathbb{R}$ is a function on the surface, then $\overline{\mathbf{g}}=e^{u} \mathbf{g}$ is another Riemannian metric of $S$. The angles on the surface measured by $\mathbf{g}$ are equal to those by $\overline{\mathbf{g}}$. Therefore, we say the metric $\overline{\mathbf{g}}$ is conformal to the metric $\mathbf{g}$, meaning angle preserving. Although there is no angle distortion, the areas are scaled, and the area distortion factor is $e^{2 u}$, which is called conformal factor. The Gaussian curvature also changes accordingly,

$$
\bar{k}=e^{-2 u}(k-\Delta u),
$$

where $\Delta$ is the Laplace-Beltrami operator induced by the original metric g. But the total curvature is preserved, which is solely determined by the topology (Gauss-Bonnet formula), $\int_{S} k d s=\int_{S} \bar{k} d \bar{s}=2 \pi \chi(S)$, where $\chi(S)$ is the Euler characteristic of the surface $S$.

All Riemannian metrics on a surface can be classified by the conformal equivalence relation. Each class is called a conformal structure of the surface. According to the uniformization theorem, each conformal structure has a unique representative, which is a Riemannian metric with constant Gaussian curvature. Therefore the surface can be locally isometrically mapped into the sphere, the plane or the hyperbolic plane respectively.

Theorem 1 (Poincaré-Koebe Uniformization). For an arbitrary closed surface with a Riemannian metric $\mathbf{g}$, there is a metric $\overline{\mathbf{g}}$ conformal to $\mathbf{g}$, whose Gaussian curvature $\bar{k}$ is $+1,0$ or -1 if $\chi(S)$ is positive, zero or negative respectively.

Such a metric is called a uniformization metric. The metric is unique if $\chi(S)<0$. For surfaces with boundaries, the uniformization metric can be defined similarly, such that all interior points have constant Gaussian curvature, 
all boundaries become geodesics. Figures 8 and 6 show two genus zero closed surfaces which are conformal to the unit sphere. Figure 8 visualize the homeomorphism using a geometric morphing process. Figure 7 shows a genus one surface, which is conformally deformed onto the plane. Figure 9 demonstrates a genus one surface with three boundaries, it is conformally mapped to the planar domain. Figure 1 shows a genus two surface, embedded in the hyperbolic disk with its uniformization metric.

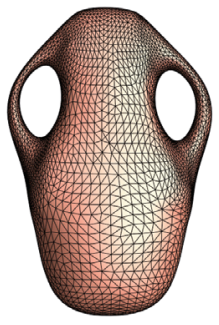

(a) Surface

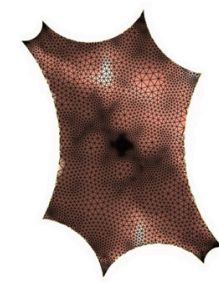

(b) Fundamental Domain(c) Embedding in hyperbolic disk

FiguRE 1. A high genus surface in (a) is conformally embedded onto the hyperbolic space shown in (c). The periods are color encoded. The shape of its period (fundamental domain) is the conformal fingerprint shown in (b).

The Ricci flow introduced by R. Hamilton [7] is a powerful tool to compute the uniformization metric.

Definition 3.1 (Surface Ricci Flow). Suppose $S$ is a closed surface with a Riemannian metric $\mathbf{g}$, the Ricci flow is defined as

$$
\frac{d \mathbf{g}}{d t}=-2 k \mathbf{g}
$$

Surface Ricci flow deforms a Riemannian metric $\mathbf{g}$ to metrics $e^{2 u(t)} \mathbf{g}$ conformal to $\mathbf{g}$. The Ricci flow (1) can be reformulated as $\frac{d u(t)}{d t}=-2 k(t)$.

The following theorems gurantee that the Ricci flow will deform a metric to the uniformization metric,

Theorem 2 (Ricci Flow). Suppose $S$ is a closed surface with a Riemannian metric $\mathbf{g}$, the normalized Ricci flow which preserves area

$$
\int d s_{\mathbf{g}(t)} \equiv C
$$


422 Junfei Dai, Wei Luo, Min Zhang, Xianfeng Gu and Shing-Tung Yau

converges. The limit metric $\mathbf{g}(\infty)$ is conformal to $\mathbf{g}$, and the limit Gaussian curvature is

$$
k(\infty) \equiv \frac{2 \pi \chi(S)}{C}
$$

Suppose the desired target curvature $\bar{k}$ is given, then the corresponding conformal metric can be achieved by the following general Ricci flow $\frac{d u(t)}{d t}=2(\bar{k}-k(t))$. Ricci Flow on Three Dimensional Manifolds. The uniformization theorem for surfaces states that any surface can be equipped with one of the three canonical geometries, spherical, Euclidean or hyperbolic geometry. Thurston's Geometrization conjecture is an analogy to it, which claims that any 3-manifold can be uniquely partitioned, and each component can be equipped with one of the eight canonical geometries.

In the case of surfaces, a special case of the uniformization theorem is for topological sphere: all compact simply connected surface is conformal to the unit sphere. The analogy in 3-manifolds is the Poincare conjecture, all compact, simply connected 3-manifold is homeomorphic to the 3 -sphere. It is a special case of Thurston's conjecture.

Ricci flow on 3-manifolds is similar to Ricci flow on surface, the only difference is to replace the Gaussian curvature by Ricci curvature in equation (1). On a surface, Ricci flow deform the metric of the surface to a canonical one such that the Gaussian curvature is constant everywhere. On a 3-manifold, Ricci flow leads to a canonical metric with constant Ricci curvature. The fundamental difference is that surface Ricci flow won't lead to singularities, but 3-manifold Ricci flow will cause singularities, which requires great efforts to handle with.

In this work, we visualize Ricci flow mainly on surfaces, because it represents most of the characteristics and there exists mature computational algorithms on the discrete surfaces.

3.2. Discrete Surface Ricci Flow. Suppose $M$ is a triangular mesh, we use $(V, E, F)$ to denote its vertices, edges and faces. We denote a vertex as $v_{i}$; an edge connecting $v_{i}, v_{j}$ as $e_{i j}$ or $\left[v_{i}, v_{j}\right]$; a face formed by $v_{i}, v_{j}, v_{k}$ counter-clock-wisely as $f_{i j k}$ or $\left[v_{i}, v_{j}, v_{k}\right]$. Suppose the mesh is embedded in $\mathbb{R}^{3}$, it has the induced Euclidean metric $g$. We use $\partial M$ to represent the boundary of the mesh.

The Riemannian metric on a mesh is determined by the edge length, therefore we use edge length function $L: E \rightarrow \mathbb{R}^{+}$to represent the discrete metric. The 
only requirement for the discrete metric is that the triangle inequality is held on all faces of the mesh.

The discrete metric can be treated as edge lengths, therefore, determines the three interior angles of each face. The discrete curvature at each vertex is a measurement of the flatness of the neighborhood of the vertex. Suppose $v$ is a interior vertex $v \notin \partial M$, then the discrete curvature at $v$ is defined as $k(v)=$ $2 \pi-\sum_{k} \alpha_{k}$, where $\alpha_{k}$ 's are the interior angles surrounding $v$. If $v$ is a boundary vertex $v \in \partial M$, then the curvature is $k(v)=\pi-\sum_{k} \alpha_{k}$. The total discrete curvature is solely determined by its topology $\sum_{v} k(v)=2 \pi \chi(M)$, where $\chi(M)$ is the Euler characteristic of $M$.

In order to compute conformal deformation of the metrics and the Ricci flow, we adopt the circle packing metric. We associate a circle with each vertex. Each face has three circles and they intersect one another. The edge length is determined by the circle radii and the intersection angle among them.

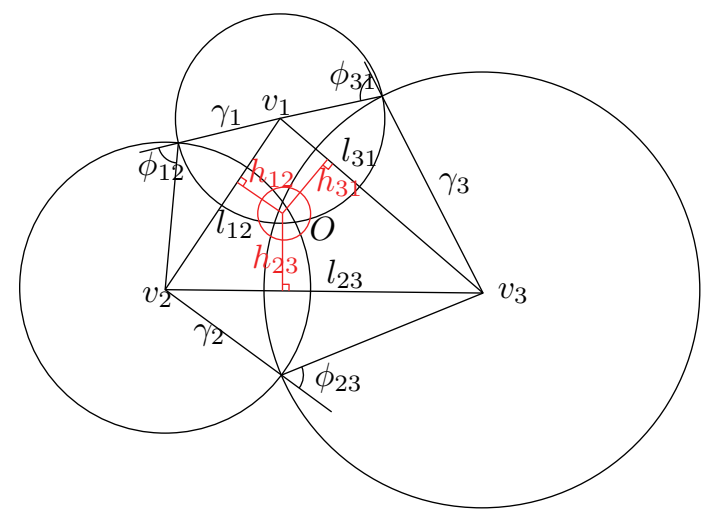

FiguRE 2. Circle packing metric for a triangle.

Definition 3.2 (Circle Packing Metric). Suppose $M$ is a triangular mesh with a circle packing, the vertex radius function is denoted as $\Gamma: V \rightarrow \mathbb{R}^{+}$, the edge angle function is denoted as $\Phi: E \rightarrow[0, \pi)$. The circle packing metric $(M, \Gamma, \Phi)$ is a discrete metric, the length of edge $e_{i j}$ is defined as

$$
l_{i j}=\sqrt{\gamma_{i}^{2}+\gamma_{j}^{2}+2 \gamma_{i} \gamma_{j} \cos \phi_{i j}},
$$

where $\gamma_{i}$ is $\Gamma\left(v_{i}\right), \phi_{i j}$ is $\Phi\left(e_{i j}\right)$. Figure 2 depicts the details. 
424 Junfei Dai, Wei Luo, Min Zhang, Xianfeng Gu and Shing-Tung Yau

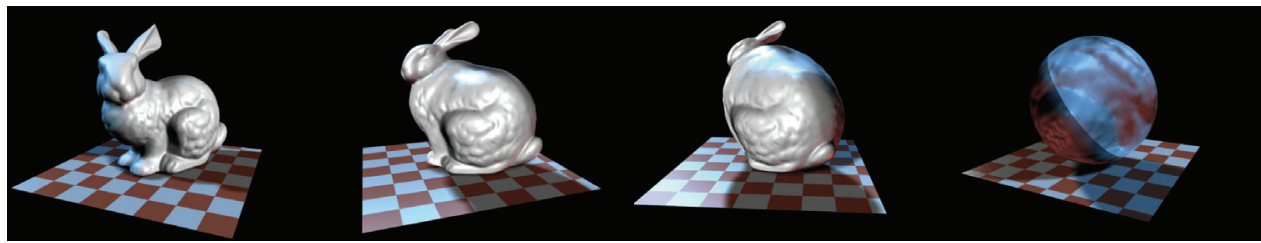

FIGURE 3. Simply connected surface without boundary is homeomorphic to the unit sphere.

Similar to the smooth case, discrete conformal deformations only change the circle radii and preserve the intersection angles. Two circle packing metrics $\left(M, \Gamma_{1}, \Phi_{1}\right)$ and $\left(M, \Gamma_{2}, \Phi_{2}\right)$ are conformal, if and only if $\Phi_{1} \equiv \Phi_{2}$. The discrete Ricci flow can be defined in the similar way as equation (1),

Definition 3.3 (Discrete Ricci Flow). Given a triangular mesh $M$ with a circle packing metric $(M, \Gamma, \Phi)$, the discrete Ricci flow is

$$
\frac{d \gamma_{i}(t)}{d t}=-2 k_{i} \gamma_{i}(t)
$$

where $k_{i}$ is the discrete curvature at the vertex $v_{i}$. In Chow and Luo's work [3], it is proved that the normalized discrete Ricci flow is convergent, and the limit discrete curvature is constant.

The discrete conformal factor is defined as $u_{i}=\log \gamma_{i}, \mathbf{u}=\left(u_{i}\right)$. Given a prescribed target curvature $\bar{k}_{i}, \overline{\mathbf{k}}=\left(\bar{k}_{i}\right)$, if there exists a discrete conformal metric with the curvature $\bar{k}$, it can be obtained by the general discrete Ricci flow $\frac{d u_{i}(t)}{d t}=$ $\bar{k}_{i}-k_{i}(t)$.

\section{Visualization of Curvature Flow}

During the Ricci flow, the curvatures in a given surface are redistributed driven by the metric deformation like a heat diffusion process. The curvature flow can be modeled as an electric network model.

Suppose the change of the curvature is $d \mathbf{k}=\overline{\mathbf{k}}-\mathbf{k}$, which is treated as the electric potential. The conductance of an edge $e_{i j}$ is denoted by $w_{i j}$, which is defined as $w_{i j}=\frac{h_{i j}^{k}+h_{j i}^{p}}{l_{i j}}$, where $l_{i j}$ is the edge length of $e_{i j}$ under metric $\mathbf{u}, h_{i j}^{k}, h_{j i}^{p}$ are the distances to $e_{i j}$ of the circumcenters of the two faces adjacent to $e_{i j}$. Then the curvature flow along edge $e_{i j}$ from $v_{i}$ to $v_{j}$ is

$$
w_{i j} \nabla d \mathbf{k}=w_{i j}\left(d k_{j}-d k_{i}\right) .
$$




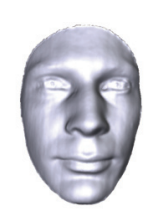

(a)

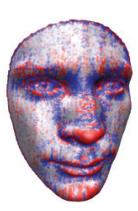

(b)

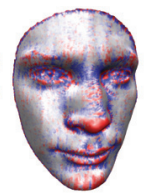

(c)

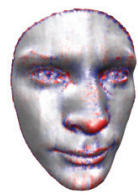

(d)

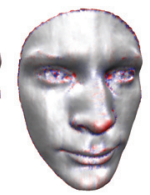

(e)

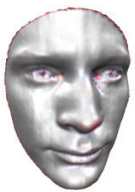

(f)

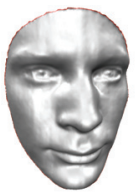

(g)

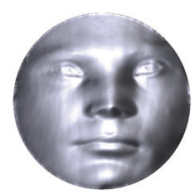

(h)

Figure 4. (a): a face model, (b)-(g): the diffusion of curvatures during the Ricci flow process. The red, blue, and gray colors indicate high, low, and zero curvatures, respectively. (g): the face model with the deformed Riemannian metric from the Ricci flow, which can be flatten in to $R^{2}$.

Figure 2 depicts the details for the configuration. We visualize the curvature flow using this electric network model.

Curvature flow as a Heat Diffusion. The curvature evolves like a heat diffusion, $\frac{d \mathbf{k}}{d t}=-\Delta(\mathbf{u}) \mathbf{k}$, where $\Delta(\mathbf{u})$ is the discrete Laplace-Beltrami operator. The elements $\Delta_{i j}=w_{i j}, i \neq j$ if $e_{i j}$ is an edge, otherwise $\Delta_{i j}$ is zero. $\Delta_{i i}=-\sum_{j \neq i} w_{i j}$.

The most simple way to visualize the behavior of the Ricci flow is the variation of the curvature values with a color spectrum during the process. Fig. 4 illustrates the curvature evolutions in a face model with Ricci flow. We set the target curvature values for the face model such that $k_{i} \equiv 0$ for the interior vertices and $\sum k_{i}=2 \pi$ for the boundary vertices. As shown in Fig. 4 (b)-(g), during the Ricci flow the high curvature values gradually decreased and the low curvature values gradually increased with the diffusions. Finally, the only boundary vertices have positive curvatures with the sum of $2 \pi$, and each interior vertex has the zero curvature (see Fig. $4(\mathrm{~g})$ ). From the target Riemannian metric, we can embed the face model onto the unit disk as shown in Fig. 4(h).

Curvature flow as the gradient field. The more intuitive way is to visualize the streams of the curvature flows on the given mesh during the Ricci flow. From this, we can clearly see the fact that the curvature flows from one place to to another place. The network of the curvature flows can be easily computed using the formulae (3).

Fig. 9 depicts the hypersheet with the curvature flows streaming from the sources to the sinks. Under the initial metric, the hypersheet model is a minimal surface, therefore, it has negative Gaussian curvature everywhere, the geodesic curvatures along the boundaries are positive. Under the final metric, the interiors 
are flat, the geodesic curvature on boundaries are negative. Therefore, as shown in Fig. 9, every circular boundaries on the hypersheet emits their curvatures and all the interior points inhale the curvatures during the Ricci flow process.
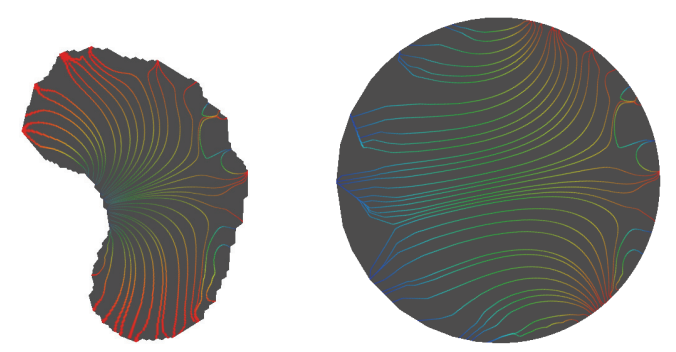

Figure 5. The curvature flow for the conformal deformation of the planar shape is shown in Figure 12. The curvature flows from red to blue.

To render the streams of the curvature flow, we utilize the texture coordinates which can be obtained with uniformization metrics. Given the $\nabla \mathbf{u}$ in each time, we compute the gradient vector in the texture domain. To visualize the flow with the gradient field, we use the image-based flow visualization [14] in the texture domain. Finally, we map the flow textures to the original mesh. Figure 5 and Figure 9 (d) illustrate the curvature flow using this method. The curvature flows from red to blue.

\section{Visualization of Conformal Structure}

The surface Ricci flow deforms a metric preserving the conformal structure. The conformal structure of a surface is an abstract concept and difficult to perceive. We visualize the conformal structure of a surface by two methods.

Canonical Embedding. Within each conformal structure of the surface, the uniformization metric is the representative, which induces a unique embedding on the sphere, the Euclidean plane or the hyperbolic plane. Therefore, the conformal structure can be visualized by this kind of canonical embedding. For example, Figure 6 shows the brain surface is conformal to the unit sphere. On the sphere, we use the normal map from the original brain surface, therefore, the correspondence can be easily conveyed by comparing the local shading pattern. 
It is also evident that, the local shapes are well preserved, which is one prominent characteristics of the conformal map.

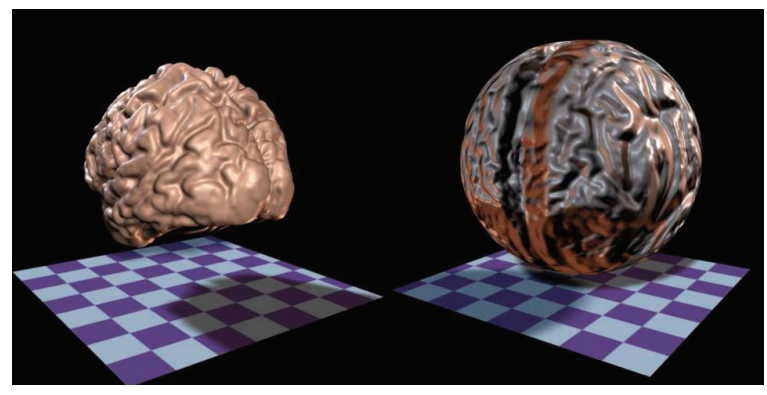

Figure 6. A Brain surface is conformal to a unit sphere.

For surfaces with complicated topologies, the canonical embedding conveys the complete information of the conformal structure. For example, for the hypersheet surface, it is of genus one with three boundaries. With its uniformization metric, it can be embedded onto the Euclidean plane periodically, each period is a parallelogram with three circular holes, the centers and the radius of the circles, the shape of the parallel gram are the fingerprint of the conformal structure.

Potential Fields on Surfaces. The conformal structure of a surface can be depicted by the potential field on the surface. The equi-potential lines and the gradient lines are orthogonal everywhere, they form a canonical grids on the surface. Figure 8 illustrates the conformal structure on the bunny surface, the red curves are equipotential lines, and the blue curves are the gradient lines. Equivalently, the grids formed by the equipotential lines and the gradient lines can be replaced by a checkerboard pattern. Figure 9 illustrates the conformal structure of the minimal surface model.

\section{Visualization of Riemannian Metrics by Embedding}

The result of Ricci flow is a Riemannian metric on the surface. It is very challenging to visualize a Riemannian metric. If the Gaussian curvature is positive everywhere, the metric determines an embedding in $\mathbb{R}^{3}$ as a convex surface unique up to rigid motion. Therefore, the metric can be visualized directly by the embedding. In discrete case, the uniqueness of the embedding is first proved by Cauchy for 2-spheres and by Alexandrov for disks. 
428 Junfei Dai, Wei Luo, Min Zhang, Xianfeng Gu and Shing-Tung Yau
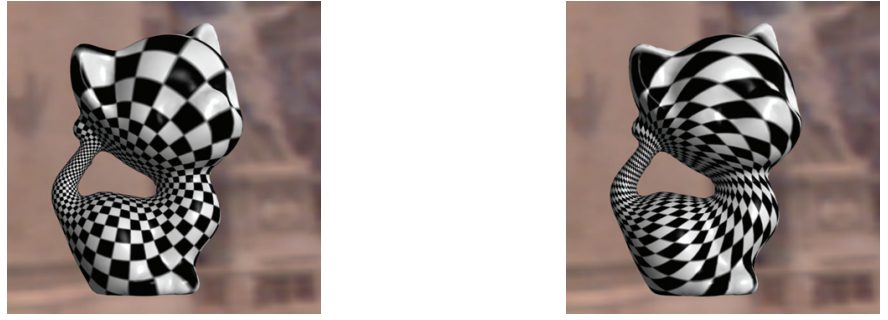

(a) Conformal texture mapping(b) Non conformal texture mapping
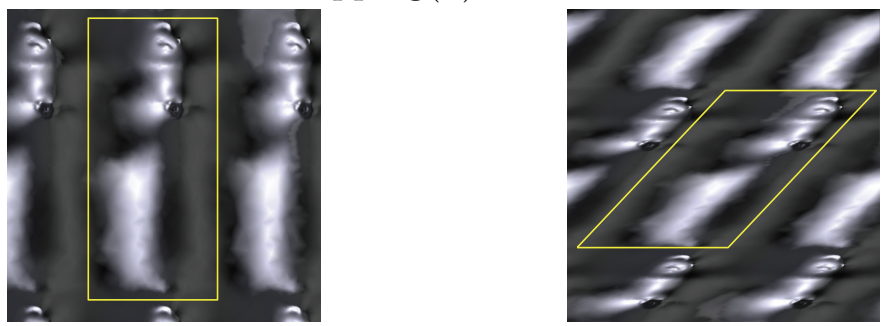

(c) Fundamental domain

(d) Skewed fundamental domain

Figure 7. The genus one surface (a) is periodically, conformally embedded onto the plane. Each period is a parallelogram (c). The shape of it is determined by the conformal structure. If the period is deformed $(\mathrm{d})$, the induced texture mapping is not conformal (b)

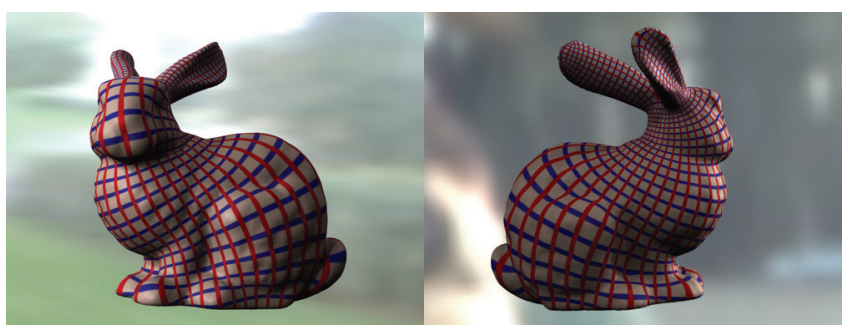

FiguRE 8. Conformal structure on the bunny surface is shown as a potential field.

A convex cap is the graph of a piecewise linear function over a convex polygonal region in the plane so that the value of the function is zero in the boundary.

Theorem 3 (Alexandrov). Let $M$ be a topological disk with a convex Euclidean polyhedral metric, there exists a convex cap $C \subset \mathbf{R}^{3}$ with the upper boundary isometric to $M$. Besides, $C$ is unique up to a rigid motion. 


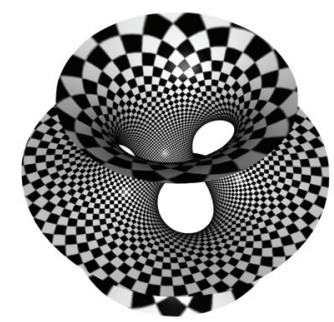

(a) Front view

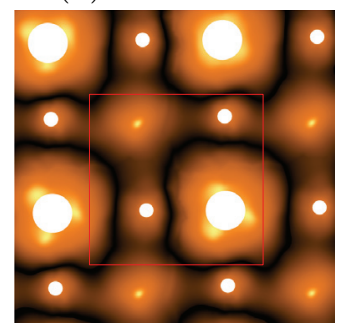

(c) Fundamental Domain

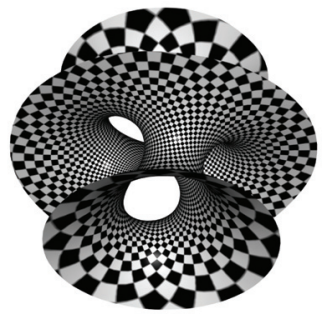

(b) Back view

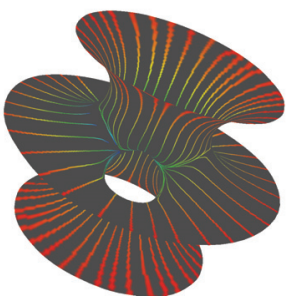

(d) Curvature Flow

Figure 9. Conformal structure on the hyper-sheet surface is shown as a potential field in (a) and (b). The surface is embedded onto the plane using uniformization metric, the three boundaries are mapped to circles (c). Curvature flow is shown in (d), the curvature flows from the red to the blue.

In his seminal theoretic work [8], Izemestiev proposed a variational method to determine the embedding. Our algorithms are based on his theoretic discovery.

Suppose $M$ is a topological disk, represented as a mesh, then each vertex $v_{i}$ is embedded in $\mathbb{R}^{3}$, the height function is $h$. The boundary vertices are on the $x y$ plane, therefore heights are zeros for all boundary vertices. There exists a unique height function, which isometrically embeds $M$ as a convex cap.

For each triangle of the mesh, the three edge lengths and the heights determine a prism, therefore all the dihedral angles along the edges can be determined. Algorithm 1 gives the details of computing the dihedral angles. The edge lengths, heights and the dihedral angles on all the prisms determine a energy, further on, this energy is defined by the height function. The energy is strictly concave. The height function, which reaches the global maximum of the energy, corresponds to the unique embedding. 
430 Junfei Dai, Wei Luo, Min Zhang, Xianfeng Gu and Shing-Tung Yau
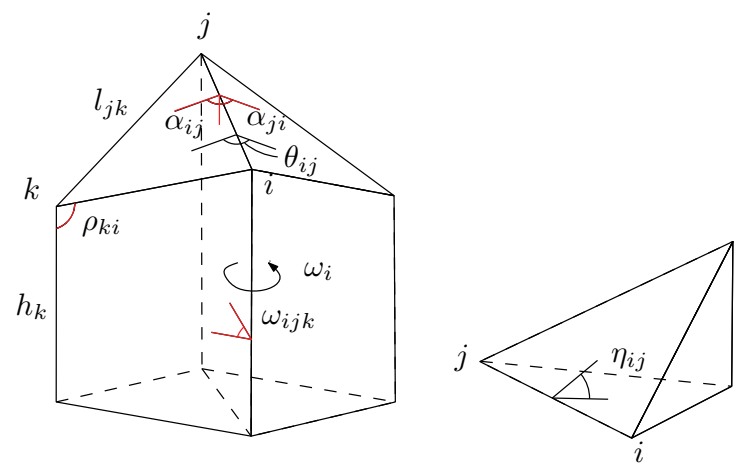

Figure 10. Total scalar function of a convex cap.

Definition 6.1 (Izemestiev). Let $C$ be a convex cap represented by $(T, h)$. The total scalar curvature of $C$ is defined as

$$
S(C)=\sum_{\Sigma / \partial D} h_{i} k_{i}+\sum_{i n t D} l_{i j}\left(\pi-\theta_{i j}\right)+\sum_{\partial D} l_{i j}\left(\frac{\pi}{2}-\eta_{i j}\right)
$$

where $k_{i}=2 \pi-\omega_{i}$ is the curvature at the $i$-th height of the cap $C, l_{i j}$ is the length of the edge $e_{i j} \in T, \theta_{i j}$ is the total dihedral angle at an interior edge $e_{i j}$, and $\eta_{i j}$ is the dihedral angle at a boundary edge $e_{i j}$, see Figure 10.

$$
\theta_{i j}=\alpha_{i j}+\alpha_{j i}, \omega_{i}=\sum_{f_{i j k} \in T} \omega_{i j k} \text {. Furthermore, the gradient of } h \text { equals }
$$

$$
\frac{\partial S}{\partial h_{i}}=k_{i}, \nabla S=\left(k_{1}, k_{2}, \cdots, k_{n}\right)^{T} .
$$

Theorem 4 (Izemestiev). The total scalar curvature $S$ is strictly concave. The height function $h$ corresponding to the global maximum induces the unique embedding.

Suppose a topological disk $D$ is given, with a discrete metric $l$, such that all curvatures are positive. We want to compute an embedding of $(D, l)$ in $\mathbb{R}^{3}$. Equivalently, we want to find a height function $h$ of $D$ to realize the mesh. $h$ can be obtained by maximizing the total scalar curvature $S$.

First, we consider the case of one triangle $T_{i j k}$, suppose the edge lengths $l_{i j}$ and vertex heights $h_{i}$ are given, the following simple algorithms realize it as a prism in $\mathbb{R}^{3}$ and compute all the dihedral angles,

Next, we consider the whole mesh, and compute the embedding with the given metric, 

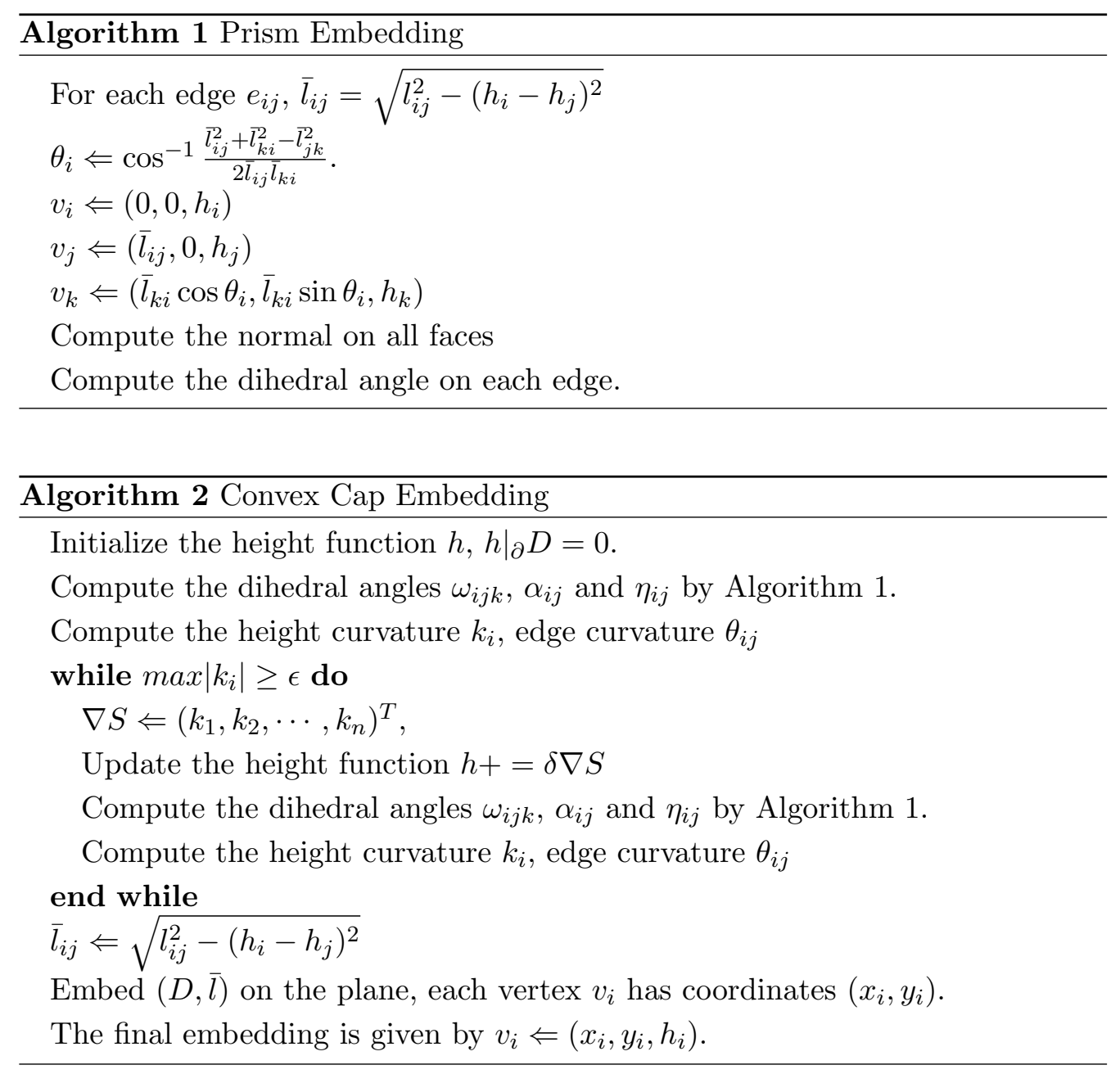

In practice, it is critical to choose the appropriate initial height functions. If all the curvature of interior vertices are zeros, the convex cap embedding is degenerated to planar case. We first embed one triangle onto the plane using the edge lengths, then embed all the faces sharing one edge with it. Then we keep growing the embed area until all the faces are flattened onto the plane.

In our application, we aim at visualizing the whole process of conformal deformation the Riemannian metric, therefore, we need to compute a sequence in order to improve the efficiency, we reuse the current embedding result as the initial guess for the next embedding. The Algorithm 3 describes the construction of a conformal deformation process in details. Suppose the input is a simply 
432 Junfei Dai, Wei Luo, Min Zhang, Xianfeng Gu and Shing-Tung Yau

connected mesh, with a single boundary, also the target curvature $\mathbf{k}_{1}$ is given, such that all curvatures are positive.

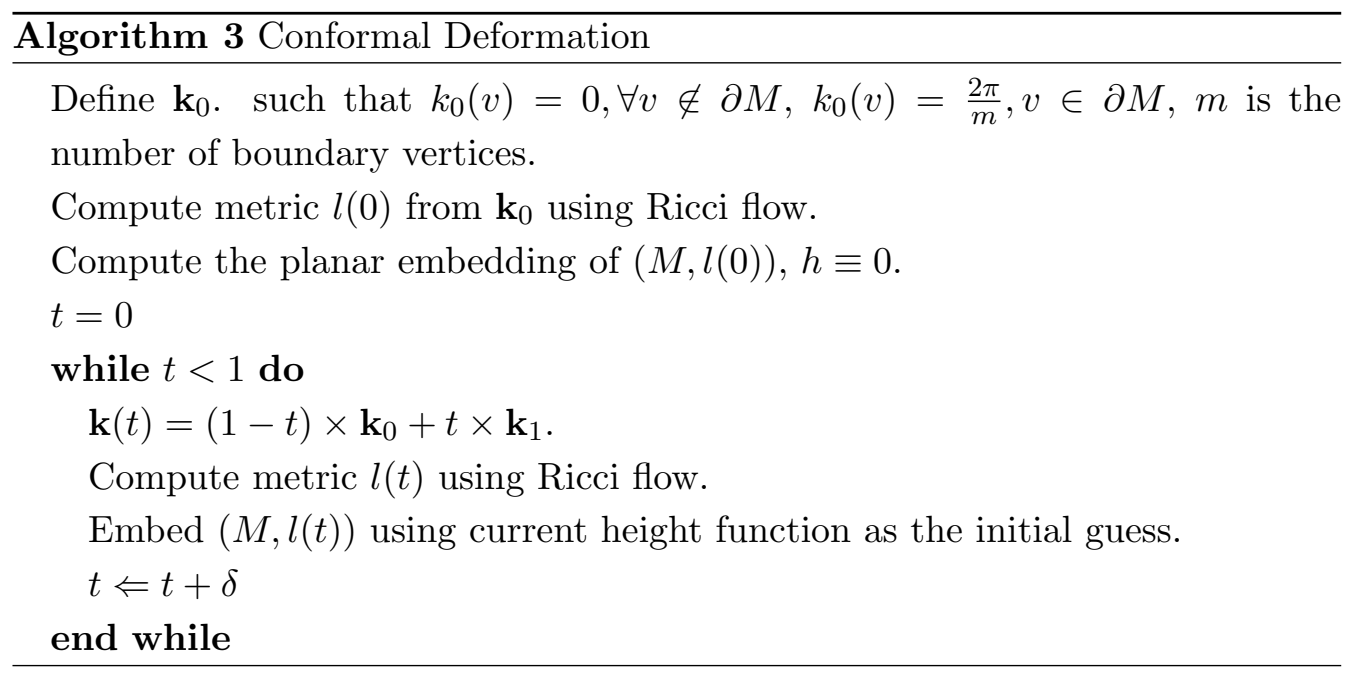

Figure 12 demonstrates a conformal deformation of a planar surface. The curvature flow from boundary to the boundary across the surface. Because the interior vertices are always flat, there is no source or sink in the interior part. Namely, the curvature flow is divergence free.

Figure 13 demonstrates a conformal deformation of a convex cap surface. The conformality is represented by checkerboard texture mapping.

\section{Visualization of the Proof of Poincaré-Koebe's uniformization THEOREM}

We visualize the proof of the uniformization theorem, as a 2-dimensional counterpart of the recent resolution of the Poincaré conjecture using Ricci flow. Namely, we want to visualize the proof of the following proposition using Ricci flow: any closed simply closed surface is homeomorphic to a sphere(see Fig. 3).

Suppose $M$ is a closed, simply connected mesh,

(1) Partition $M$ to $M_{1}$ and $M_{2}$, such that $\gamma=M_{1} \bigcap M_{2}$ is a simple closed curve on $M$, both $M_{1}$ and $M_{2}$ are simply connected with a single boundary.

(2) Set the target curvature of $M_{i}$ as $\mathbf{k}_{i}$, such that

$$
\mathbf{k}_{i}(v)=0, \forall v \in \partial M_{i},
$$



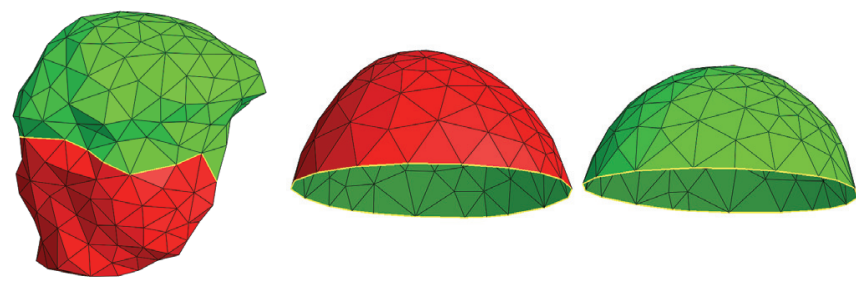

FiguRE 11. Illustration of the proof of Poincaré-Koebe theorem. The original surface is partitioned to two parts. Ricci flow is run on each component, such that all curvatures are non-negative. Each component is embedded to a convex cap, which is topologically equivalent to a hemisphere.

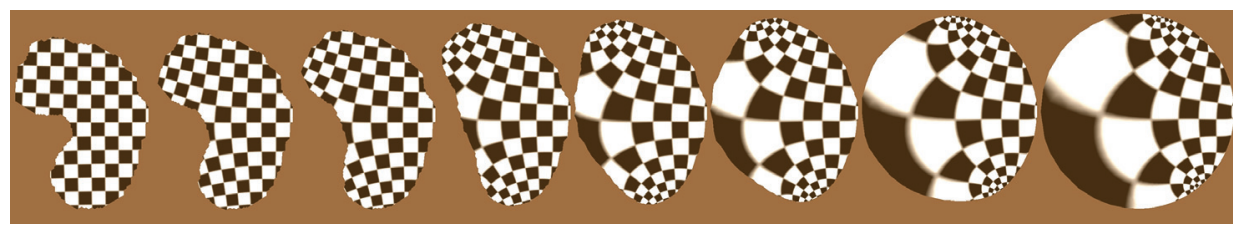

Figure 12. Conformal Deformation of a planar shape. The curvature flows from boundary points to boundary points. The deformation preserves the conformal structure.

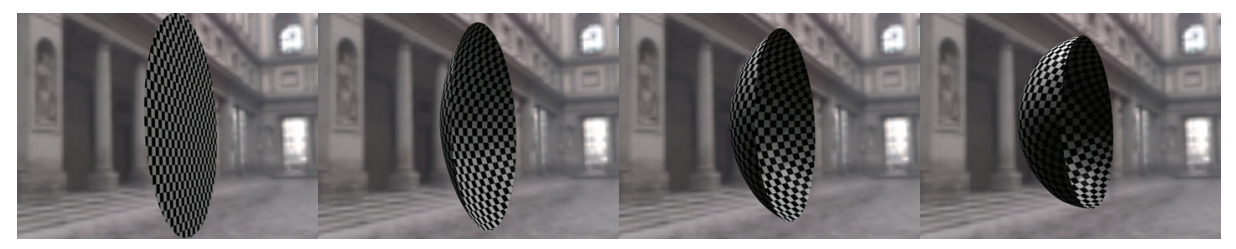

Figure 13. Conformal Deformation of a convex cap surface.

and

$$
\mathbf{k}_{i}(v)=\frac{2 \pi}{m_{i}}, m_{i}=\left|\partial M_{i}\right|, \forall v \notin \partial M_{i},
$$

(3) Use Ricci flow to compute the desired metric $l_{i}$, which induces $\mathbf{k}_{i}$.

(4) Embed $\left(M_{i}, l_{i}\right)$ using the convex cap embedding algorithm.

(5) The embedded $M_{i}$ is homeomorphic to the semi-sphere using Gauss map. 
434 Junfei Dai, Wei Luo, Min Zhang, Xianfeng Gu and Shing-Tung Yau

\section{Conclusion}

This work introduces a set of algorithms to visualize the intrinsic geometric curvature flow, Ricci flow, on surfaces. The algorithms for visualizations of conformal structures and conformal metric deformations are also discussed in details. This work presents a novel algorithm for embedding convex cap using variational approach based on recent theoretic discovery by Izmestiev [8]. As an application, the schematic proof of Poincaré conjecture using Ricci flow is illustrated for the 2 dimensional analogy.

In the future, we will generalize the discrete surface Ricci flow to three dimensional manifolds and explore embedding algorithms from Riemannian metrics for general surfaces.

\section{REFERENCES}

[1] A. Bobenko and P. Schröoder. Discrete willmore flow. In Symposium on Geometry Processing, pages 101-110, 2005.

[2] A. I. Bobenko and B. A. Springborn. Variational principles for circle patterns and koebe's theorem. Transactions of the American Mathematical Society, 356:659-689, 2004.

[3] B. Chow and F. Luo. Combinatorial ricci flows on surfaces. Journal Differential Geometry, 63(1):97-129, 2003.

[4] M. S. Floater and K. Hormann. Surface parameterization: a tutorial and survey. In Advances in Multiresolution for Geometric Modelling, pages 157-186. Springer, 2005.

[5] X. Gu, Y. He, M. Jin, F. Luo, H. Qin, and S.-T. Yau. Manifold splines with single extraordinary point. In ACM Symposium on Solid and Physics Modeling, pages 61-72, 2007.

[6] X. Gu, Y. He, and H. Qin. Manifold splines. Graphical Models, 68(3):237-254, 2006.

[7] R. S. Hamilton. Three manifolds with positive ricci curvature. Journal of Differential Geometry., 17:255-306, 1982.

[8] I. Izmestiev. A variational proof of alexandrov's convex cap theorem. Technical Report arXiv.org, March 062007.

[9] M. Jin, F. Luo, and X. Gu. Computing surface hyperbolic structure and real projective structure. In ACM Symposium on Solid and Physics Modeling, pages 105-116, 2006.

[10] L. Kharevych, B. Springborn, and P. Schröder. Discrete conformal mappings via circle patterns. ACM Transactions on Graphics, 25(2):412-438, 2006.

[11] A. Sheffer, E. Praun, and K. Rose. Mesh parameterization methods and their applications. Foundations and Trends ${ }^{\circledR}$ in Computer Graphics and Vision, 2(2):105-171, 2006.

[12] K. Stephenson. Introduction To Circle Packing. Cambridge University Press, 2005.

[13] W. P. Thurston. Geometry and Topology of Three-Manifolds. Princeton lecture notes, 1976. 
[14] J. J. van Wijk. Image based flow visualization. In SIGGRAPH '02: Proceedings of the 29th annual conference on Computer graphics and interactive techniques, pages 745-754, New York, NY, USA, 2002. ACM Press.

Junfei Dai

Zhejiang University

E-mail: jfdai@zju.edu.cn

Wei Luo

Zhejiang University

E-mail: luowei@cms.zju.edu.cn

Min Zhang

Stony Brook University

E-mail: mzhang@cs.sunysb.edu

Xianfeng $\mathrm{Gu}$

Stony Brook University

E-mail: gu@cs.stonybrook.edu

Shing-Tung Yau

Harvard University

E-mail: yau@math.harvard.edu 